This item was submitted to Loughborough's Research Repository by the author.

Items in Figshare are protected by copyright, with all rights reserved, unless otherwise indicated.

\title{
Influence of short rear end tapers on the base pressure of a simplified vehicle
}

PLEASE CITE THE PUBLISHED VERSION

http://dx.doi.org/10.4271/2015-01-1560

PUBLISHER

(C) SAE International

VERSION

AM (Accepted Manuscript)

LICENCE

CC BY-NC-ND 4.0

REPOSITORY RECORD

Perry, Anna K., Martin A. Passmore, and Ashley Finney. 2015. "Influence of Short Rear End Tapers on the Base Pressure of a Simplified Vehicle". figshare. https://hdl.handle.net/2134/17660. 


\title{
Influence of Short Rear End tapers on the Base Pressure of a Simplified Vehicle.
}

\author{
Anna-Kristina Perry, Martin Passmore, and Ashley Finney • Loughborough Univ \\ Author, co-author (Do NOT enter this information. It will be pulled from participant tab in \\ MyTechZone) \\ Affiliation (Do NOT enter this information. It will be pulled from participant tab in MyTechZone)
}

\begin{abstract}
This paper looks into the effect on base pressure of applying a high aspect ratio chamfer to all edges of a simplified squareback model (the Windsor model). The effects are investigated using force and moment measurements along with surface pressure measurements on the slanted surface and vertical base. The work forms part of a larger study to develop understanding of the mechanisms that influence overall base pressure and hence the resulting aerodynamic drag.

A short slant (approx. 4\% of model length) was applied to the trailing edges of the simplified vehicle model, representing the small rear end optimisation typical of many real vehicle geometries. Two experiments were performed: the first applied a chamfer at varying angles to the top and bottom edges; the second test looked at the same chamfer angle applied to the sides of the model geometry while the top and bottom angle remained square. The changes in drag are discussed and explained in the context of the base pressures and area weighted pressure coefficients.
\end{abstract}

\section{Introduction}

Advances in conventional, hybrid, fuel cell and EV powertrain systems and the drive for lightweight vehicles continues to play a leading role in the reduction of vehicle emissions. However, along with proposed changes to emissions legislation these advances are placing increased importance on vehicle aerodynamics. In particular with the need to reduce drag to lower energy consumption and increase range, but also to mitigate the effects of reducing weight on the vehicle crosswind behavior.

The use of square-back geometries is popular in the European market for small hatchback vehicles, estates and SUVs because of the additional cabin or load carrying space that it allows. For such square-back vehicles the flow field is dominated by the wake that gives rise to a large suction region on the rear faces that is a major component of the aerodynamic drag. The wake structure is typified by the shear layers emanating from the vehicle sides, roof and floor, containing, in the steady state, a toroidal wake structure [1].

To reduce the vehicle drag, it is required to manipulate both the size and structure of the wake in order to affect an increase in the base pressure. This can be achieved through passive optimization, for example geometry changes, vortex generators, flaps, and surface roughness, or using active control, for example, suction, blowing, oscillated suction and blowing, moveable vortex generators or

Page 1 of 11 flaps [2-11]. Active flow control is an attractive option because of the potential freedom it allows for vehicle styling as all that is required externally is the jet orifices. However these systems require additional components, increase vehicle weight and cost and absorb energy to operate. Experimentally they have been shown to change the wake structures but there are few examples where they are shown to provide a net reduction in energy consumption that would justify their application.

There are a number of passive drag reduction and optimization studies reported in the literature and studies that describe the wake structure and its effect on the aerodynamic drag. The most widely reported is that of Ahmed [12], who describes the changes in drag and flow-field between fastback and square-back geometries. The reduction in drag demonstrated for the optimum fastback compared to a square-back and the accompanying explanation provided considerable insight and has been influential in vehicle aerodynamic design ever since.

Achieving similar drag reductions in a squareback without significantly affecting the basic squareback design might be achieved through the application of a high aspect ratio slant to the trailing edges. Littlewood \& Passmore [13] investigated this by applying a short taper to the top rear edge of a squareback geometry. The slant length was selected to be representative of what might be acceptable in a practical vehicle design and the work showed a drag minimum at $12^{\circ}$, with a reduction in $C_{D}$ of $2.7 \%$ whilst the base area has only been reduced by $1.1 \%$. By considering the contributions of each of the surfaces Littlewood \& Passmore showed that the drag reduction arises out of the increased base pressure and that above $12^{\circ}$ the suction pressures on the slanted surface and the influence of the trailing vortices outweigh the pressure increase on the vertical base surface. The flow-fields included in the paper show the rear end taper inducing a downwash from the rear surface, helping to reduce the size and length of the wake structure.

In a separate study Littlewood, Passmore \& Wood [14] considered the application of horizontal slats to the base of a squareback geometry, with the intention of disrupting the near wall flow and modifying the wake structure. At model scale this produced a drag reduction of up to $\Delta C_{D}=0.008$ and was consistent with changes to the measured base pressures. Much of the gain was in the upper region close to the upper slat. Though quite different in nature these two pieces of work show that changes to the near wake structure can alter the base pressure and reduce drag.

Most recently, Grandemange [15] has applied short trailing edge flaps to the base of the Ahmed geometry. It was found that when the 
bottom flap was at $0^{\circ}$, the optimum top flap angle was $6^{\circ}$ reducing the drag by $1.8 \%$ of the baseline case; this is just half of the optimum inclination found by Littlewood \& Passmore [13], and while the geometry is not the same, the change to the drag can also be attributed to the creation of a base region cavity, leaving a region of quiescent air in the vicinity of the base and increasing the base pressure. Grandemange showed that when the bottom flap angle remained constant, the drag coefficient was a polynomial fit with the top flap angle as the independent variable while the lift coefficient was an affine function of top flap angle. An affine function contains a linear function plus a translation of the function. The work of Grandemange, showed that there are strong interactions coming from the over- and underbody flows at affect a rear end shape optimization.

This paper focuses on the use of short trailing edge tapers but extends the work of Littlewood \& Passmore to consider top and bottom edge high aspect ratio tapers varied asynchronously over a range of angles to investigate the interaction of the over- and underbody flow when there is no base cavity present; and secondly the application of a similar taper to the vertical sides of the base. As in similar work reported in the literature a simplified vehicle geometry is used to ensure that the results can be generalized and to ensure that the results are insensitive to Reynolds number.

\section{Experimental Setup}

\section{The Facilities}

All testing was carried out in the Loughborough University Wind Tunnel, full details of which can be found in Johl, Passmore \& Render [16]. The normal operating velocity in the test section is $40 \mathrm{~m} / \mathrm{s}$ with freestream turbulence intensity of approximately $0.2 \%$ and flow uniformity of $\pm 0.4 \%$. The tunnel is not equipped with a moving ground plane and it is recognized that this is a limitation if the results are to be used in an optimization process. However here the work is designed to compare the underlying relationship between geometries and the trends are expected to be well represented.

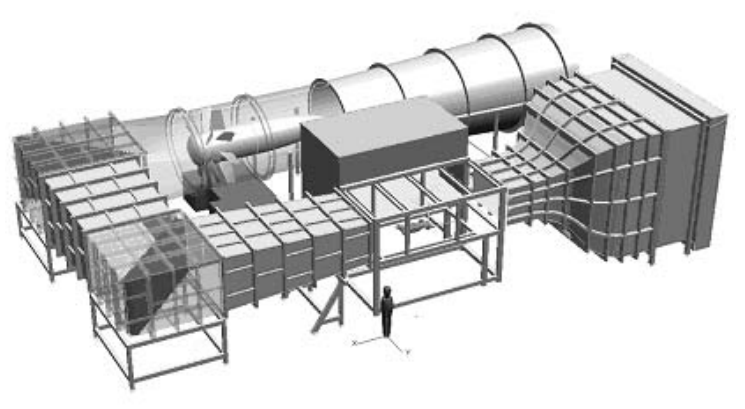

Figure 1 The Loughborough University Wind Tunnel

\section{The Model}

The Windsor Model, as used in $[17,18]$ was used in this work, its general dimensions are shown in Figure 2. The model is equivalent to an approximately quarter scale road car and gives a tunnel blockage of $4.4 \%$ in the $2.5 \mathrm{~m}^{2}$ working section. The model is designed with relatively large leading edge radiuses $(0.05 \mathrm{~m})$ to prevent local separation and the windshield to roof leading edge has a radius of $0.20 \mathrm{~m}$. The longitudinal and rear edges are all sharp.
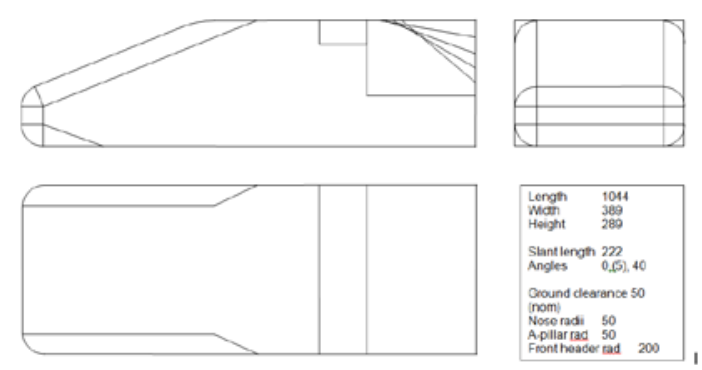

Figure 2 Basic dimensions of the Windsor Model

The model has been constructed using a machinable model board and has multiple rear inserts to implement the different rear slant angles required for this work. This is illustrated in Figure 3. For each experiment a different rear section of the model was made into which the angled inserts were located. All the tapers were $45 \mathrm{~mm}$ in length, giving an aspect ratio of 8.66. For the top and bottom taper work, slant angles of $0^{\circ}, 6^{\circ}, 12^{\circ}, 16^{\circ}$ and $20^{\circ}$ were used; for the side taper work slant angles of $0^{\circ}, 4^{\circ}, 8^{\circ}, 12^{\circ}, 16^{\circ}$ and $20^{\circ}$ were used.

The model is supported by four pins of M8 threaded bar in locations representative of front and rear axles and $10 \mathrm{~mm}$ inboard of the model sides. The loads are transferred from the model to the 6-component underfloor balance via the supporting pins. Ground clearances can be adjusted by adjusting the pins. In this work a ground clearance of $(\mathrm{h} / \mathrm{H})=0.17$ is used throughout and is typical of an MPV ride height. Based on the model overall length $(1.044 \mathrm{~m})$ the Reynolds number for this work is $2.8 \times 10^{6}$.

The MIRA co-ordinate system is used throughout this work. The origin of the system is located on the tunnel floor at mid-wheelbase, mid-track.
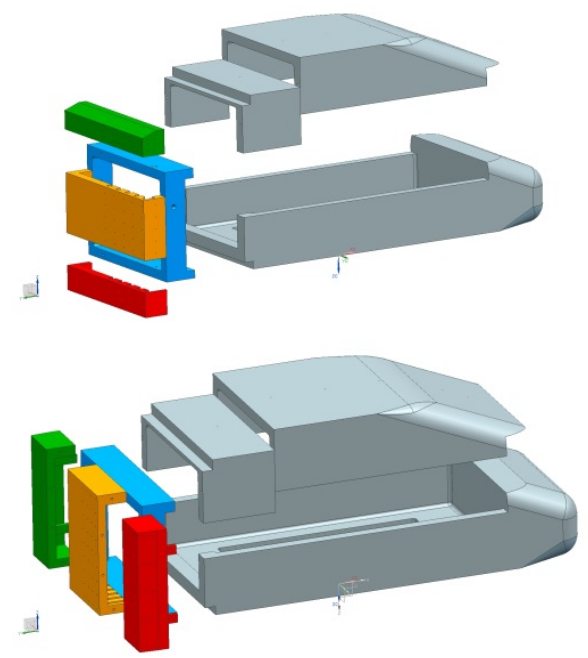

Figure 3 Exploded view of the Loughborough University Windsor Model for the two different rear end configurations 


\section{Balance Measurements}

Balance data was sampled for twenty five seconds after a ten second settling time. Prior to testing repeatability checks were performed whereby the model was set up in the tunnel multiple times and the yaw sweep results compared. The balance measurements are repeatable to $\Delta C_{x}=0.001$, which is a balance force of $0.1 \mathrm{~N}$.

\section{Pressure Measurements}

The rear facing surfaces of the model were populated with a grid of pressure tappings as shown in Figure 4. The tappings are limited to one half of the model to allow for greater resolution.

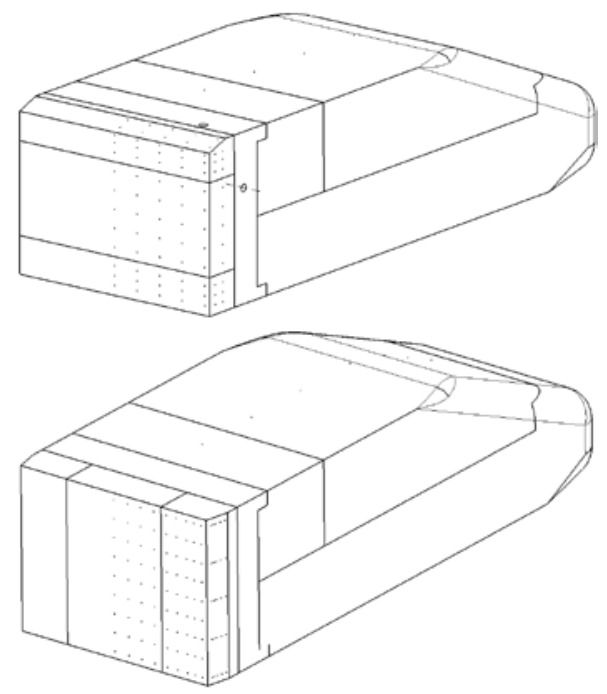

Figure 4 Distribution of surface pressure tappings on rear facing surfaces of the Windsor Model for the two different rear end configurations

The pressure measurements were made using two 64 channel miniature pressure scanners accurate to $\pm 0.15 \mathrm{~mm} \mathrm{H}_{2} \mathrm{O}$. Samples were triggered by an externally supplied $260 \mathrm{~Hz}$ signal generator and samples were taken for 31 seconds for each model configuration.

Pressure coefficients for the model surface have been calculated using the free-stream dynamic pressure (recorded at the start of the working section). All results have been blockage corrected using the MIRA blockage correction (based on continuity), Equation 1.

$C_{P_{\text {corr }}}=1-\left(\frac{1-C_{P}}{\left(1-\frac{T A}{A}\right)^{-2}}\right)$

TA - Tunnel Area

$C_{P}-$ Recorded Surface Pressure Coefficient

$C_{\text {Pcorr }}$ - Continuity Corrected Pressure Coefficient

\section{Results \& Discussion}

\section{Top and Bottom Edge Taper}

For this work, top and bottom angles of $0^{\circ}, 6^{\circ}, 12^{\circ}, 16^{\circ}$ and $20^{\circ}$ were used. These were applied asynchronously to create sweeps through each top edge taper for a given bottom edge taper giving 25 configurations. Non-yawed balance data and surface pressure measurements were collected for each configuration.

The best drag result is seen for a $16^{\circ}$ top slant angle and a $6^{\circ}$ bottom slant angle where a reduction of $\Delta \mathrm{C}_{\mathrm{d}}=-0.014$ compared to the baseline square-back is achieved (Figure 5). For all bottom slant angles greater than $6^{\circ}$ there is a marked increase in the drag of all the configurations except for the $16^{\circ}$ top slant angle with a $12^{\circ}$ bottom angle which shows a reduction of $\Delta \mathrm{C}_{\mathrm{d}}=0.003$ compared to the $16^{\circ}$ top slant angle with a $0^{\circ}$ bottom angle.

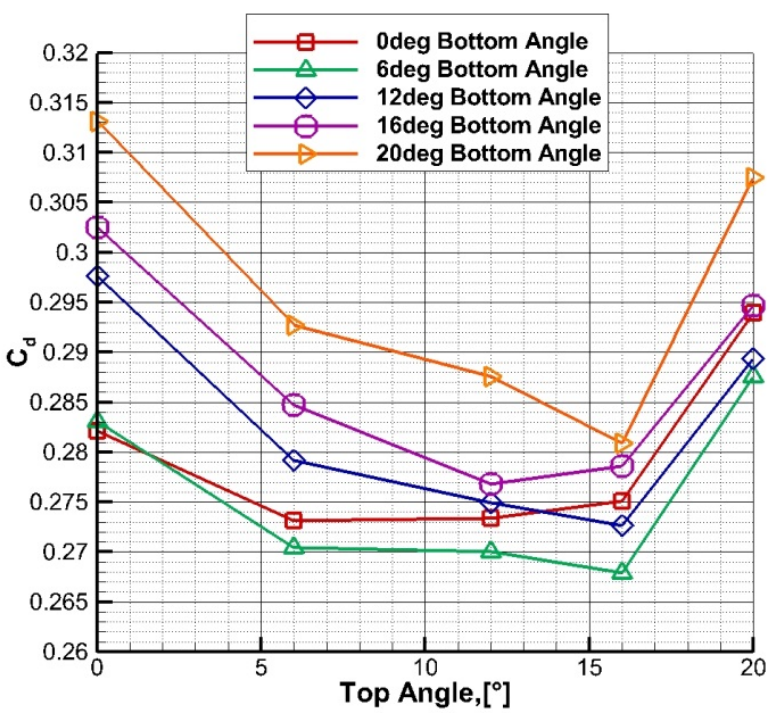

Figure 5 Top slant angle vs Drag Coefficient for all bottom slant angles

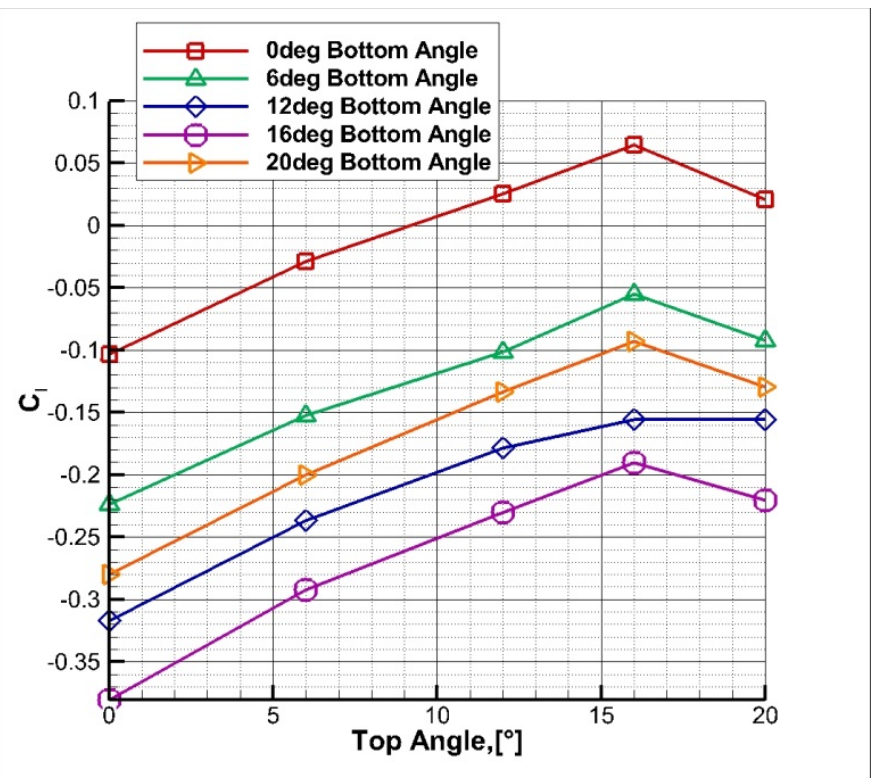

Figure 6 Top slant angle vs Lift coefficient for all bottom slant angles

All the drag results follow a similar trend and increasing the bottom slant angle leads to a shift of the data rather than fundamentally changing the shape. There is however some interaction occurring

Page 3 of 11 
between the top and bottom slant angles, which is most clearly seen in the moving local drag minimum.

For a $0^{\circ}$ top angle, the data shows that increasing bottom angle leads to an increase in drag coefficient. This is attributed to an additional up-wash from the underbody diffusing section. Looking more closely at the data suggests that the drag minimum may actually occur somewhere between $0^{\circ}$ and $6^{\circ}$, with the diffuser causing a small amount of pressure recovery that is offset by some separation on the diffusing slant angle that increases the drag back to the baseline case by $6^{\circ}$. Jowsey \& Passmore [19], at a non-dimensional ride height of $\mathrm{h} / \mathrm{H}=0.14$ (the maximum tested), found the lowest drag case was the baseline squareback shortened Ahmed model, with all diffuser angles resulting in a drag increase.

The lift results, Figure 6, show that as the top angle is changed from the $0^{\circ}$ baseline case to $16^{\circ}$ there is a monotonic increase in the lift, giving a maximum for the $16^{\circ}$ top angle for all bottom angles. For the $20^{\circ}$ top angle, there is a drop off in the lift results for all bottom angle cases except the $12^{\circ}$ case where the result is constant (within the experimental error of $\varepsilon= \pm 0.001$ ). The decrease in the lift for the $20^{\circ}$ top angle cases suggests a complete flow separation from the upper slanted surface.

Conversely, as the bottom angle is increased from $0^{\circ}$ to $16^{\circ}$ there is now a monotonic decrease in the lift for all top angles before the lift value increases again for the $20^{\circ}$ bottom angle case. The increasing lift for the $20^{\circ}$ angle case indicates flow separation from the diffuser and a loss in efficiency.

The lower slant, while only $4 \%$ of the model length is included as a means of increasing base pressure but acts somewhat like an underbody diffuser. In the literature a number of diffuser studies are reported but use considerably longer slant lengths. Both Cooper [20] and Jowsey \& Passmore [19] used 25\% diffusers with end plates and found similar monotonic decreases in the lift. Cooper's work has a maximum diffuser angle of $15.6^{\circ}$ which is also the minimum lift condition, while Jowsey \& Passmore found a lift minimum for the $16^{\circ}$ diffuser, as seen in this work. When Jowsey \& Passmore then increased the diffuser angle further, there was only a significant increase in the lift (reduction in down-force) when the diffuser angle was $25^{\circ}$ or greater. By increasing the slant length, a larger separated region may form with reattachment still occurring within the slant length consequently maintaining the advantageous diffuser up-wash characteristics, even though diffuser efficiency is reduced.

If the relationship between drag and lift is now examined more closely though plotting the two values against each other, parabolic curves can be fitted through the data, shown in Figure 7. These parabolic curves take the form of Equation 2, based on the work of Grandemange [15] and the application of induced drag coefficient theory [21]. Grandemange recorded similar results to those presented here when using trailing edge flaps rather than slants. In Equation 2 the values of $C_{D 0}$ and $C_{L 0}$ refer to the values of drag and lift coefficients for the minimum drag condition; the value of $k$ defines the gradient of the parabola. For this work the fit generates a value of $k=1.45 ; 27 \%$ larger than that found by Grandemange. This can be attributed to the lack of cavity region on the base in the present work. The presence of the flaps in the work of Grandemange act to remove the separated flow from the near wall region and leave a region of quiescent air next to the base. This is well known $[3,4,5,10]$ to create an increase in the local base pressure, and is often presented as a passive method for drag reduction on commercial vehicles. In the work of Grandemange, changing the flap angles will then act to change the wake size and energy as well as the balance between the upper and lower vortex structures and the interaction between these.

$$
C_{D}=C_{D 0}+k\left(C_{L}-C_{L 0}\right)^{2}
$$

Using the parabolic curve, an "optimum" drag and lift value can be found for each bottom angle; the "optimum" condition is taken as the apex of the parabola i.e. the minimum drag. It is noted that for each curve there is one point which does not fall close to the parabola; these are all results for the $20^{\circ}$ top angle. It is suggested that this is due to complete flow separation on the top surface resulting in a modified wake structure and therefore a drag value that no longer follows the trend. If this relationship is examined using the rear lift coefficient value, then the minimum drag value occurs at the same point in the parabola which is not necessarily the nearest zero value.

The parabolic curve fit seen in Figure 7, is known to define the vortex drag for the top angle surfaces, with some error due to base pressure changes that are caused by the downwash from the upper slant [21]. Howell \& Le Good [21] showed that for a long slant the actual vortex component of the drag matched that predicted from wing theory relatively well. That is not the case in this work where a $k$-factor of 1.45 is found against an expected value of 0.24 for this high aspect ratio slant. This is attributed to the large base area in comparison to that of the slant; the base drag therefore dominating the results.

The data can also be collapsed by normalizing using the optimum point for that data set: $\left(C_{D}-C_{D 0}\right)$ and $\left(C_{L}-C_{L 0}\right)$, as shown in Figure 8. Equation 2 can then be used to create a parabolic fit through the data using the same fit parameter of $k=1.45$. While most of the data fits on a single parabola here also the $20^{\circ}$ top angle (purple circle) results do not fit the trend. There is also a further cluster of points highlighted in the cyan circle that are for the $16^{\circ}$ top angle. This would indicate that although evidence of flow separation is not seen in the balance results (for example through a sharp increase in the drag value), there may be some change to the flow mechanisms at the rear of the model and this is shifting the data away from the general trend.

While the results for $20^{\circ}$ top angle do not match the trend, the top angle results for a $20^{\circ}$ bottom angle do lie on the parabola. This would indicate that the ground proximity of the lower slant angle is encouraging attached flow. Jowsey \& Passmore [19] found that diffuser angles of both $19^{\circ}$ and $22^{\circ}$ still work well with regards to lift generation at a ground proximity of $(\mathrm{h} / \mathrm{H})=0.14$, although separation is starting to occur at the diffuser inlet at a diffuser angle of $16^{\circ}$ and this results in a progressive decrease in the lift coefficient, as also seen here in the balance results, Figure 6 .

The surface pressures are now considered, to further explain the trends seen in the balance data. Selected results are presented in the text while a complete data set is shown in the Appendix. Figure 9 through to Figure 13 show the time averaged contour plots of the rear facing surfaces with the left hand column showing the top slant and base pressures and the right hand column showing the lower slant and repeating the base pressures. 


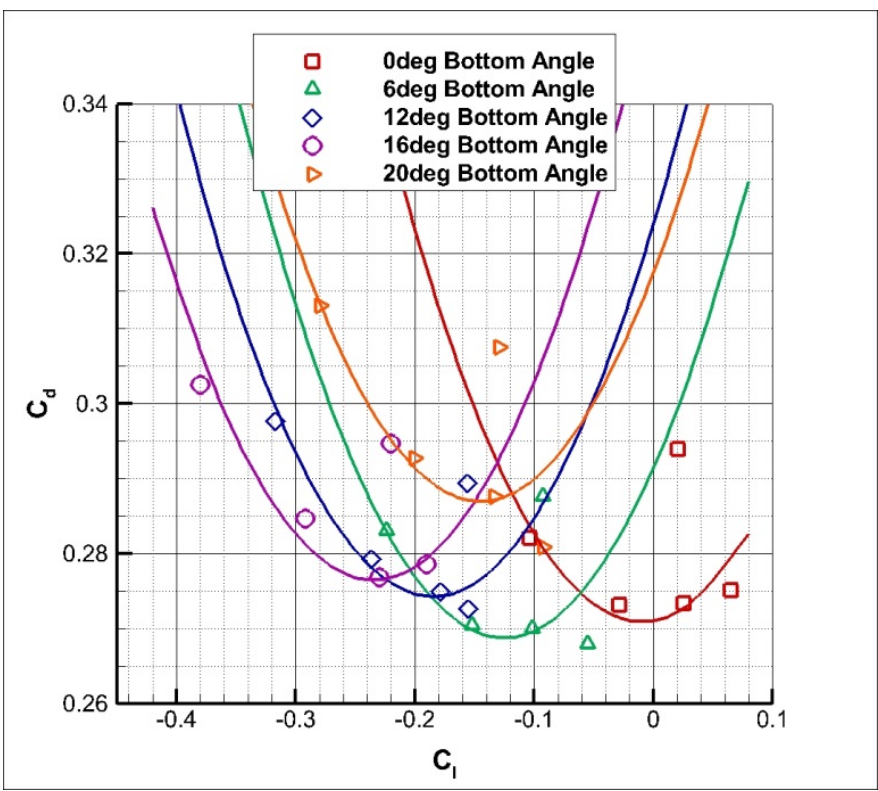

Figure 7 Drag vs Lift Coefficient for all bottom slant angles with parabolic data fit curves of $k=1.45$

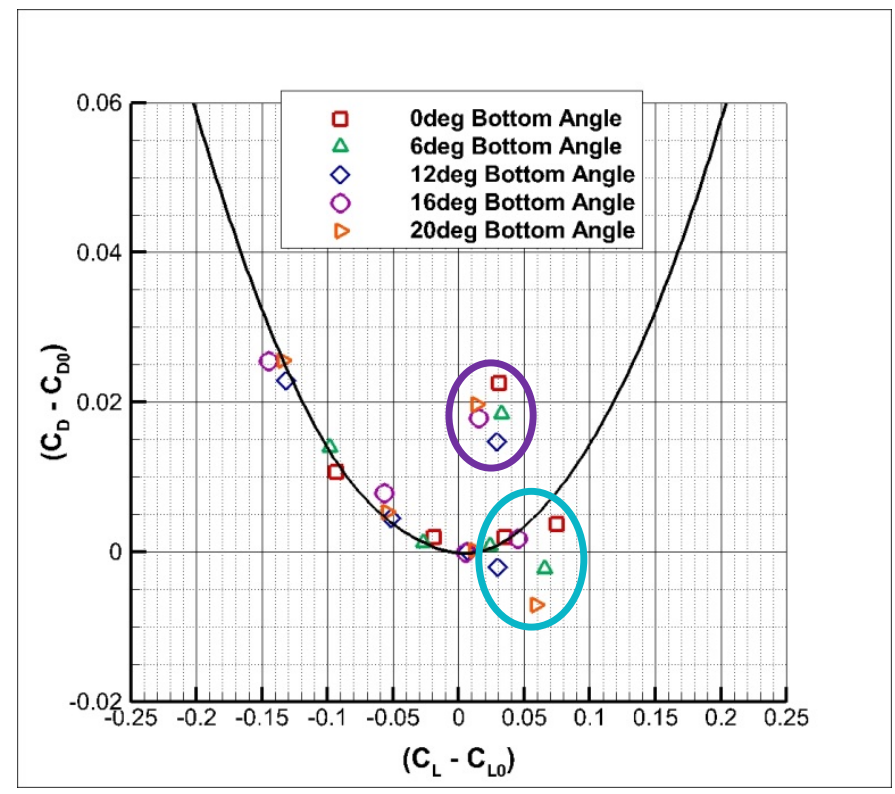

Figure 8 Reduced Drag vs Reduced Lift Coefficient with a parabolic data fit

For the $0^{\circ}$ bottom angle the balance results show that as a $6^{\circ}$ top slant angle is applied, there is an initial decrease in the drag coefficient of $\Delta C_{D}=-0.009$ and an increase in the lift coefficient of $\Delta C_{L}=0.075$ from the baseline squareback case. This is evident in Figure 9, which shows a lower pressure on the slanted surface, accounting for the lift increase, and an increased base pressure giving the drag reduction. When compared to the baseline squareback case, there is a higher pressure region that is spreading across the base from the side of the model. As the top slant angle is increased further through the $12^{\circ}$ and $16^{\circ}$ cases the drag at first remains constant (within the experimental error of $\varepsilon=0.001$ ) before increasing by $\Delta C_{D}=0.002$. The high pressure region seen in the $6^{\circ}$ case progressively spreads further over the base area until at $16^{\circ}$ the flow in the centerline has also undergone some increase in pressure.

Page 5 of 11
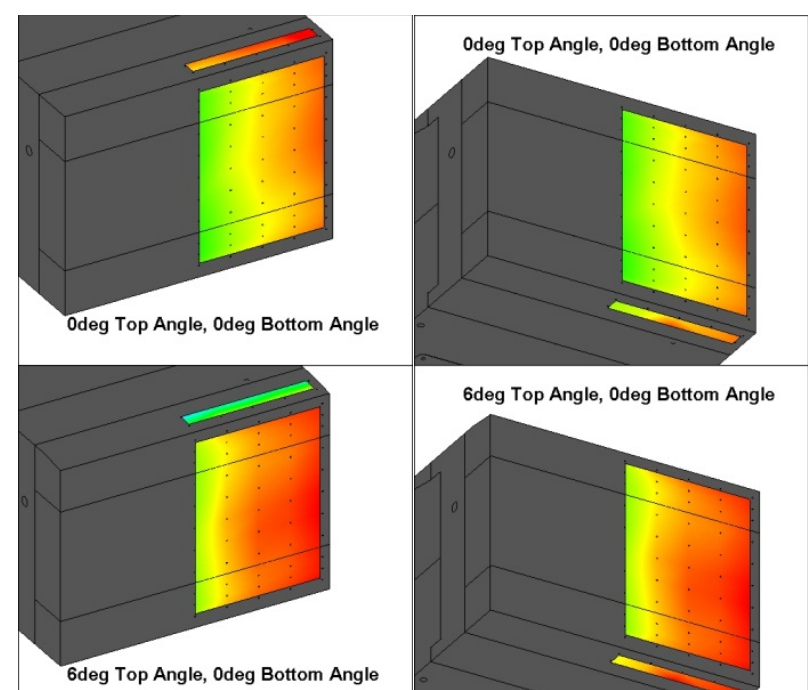

6deg Top Angle, Odeg Bottom Angle
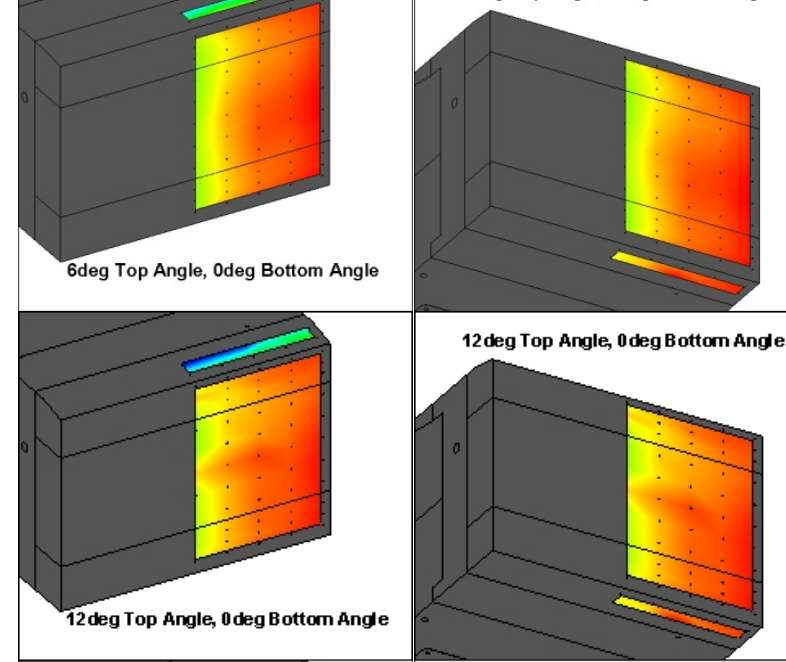

12 deg Top Angle, 0 deg Bottorn Angle

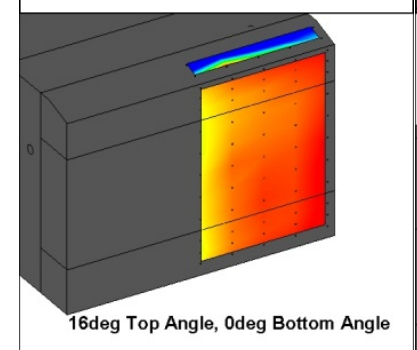

16deg Top Angle, Odeg Bottom Angle
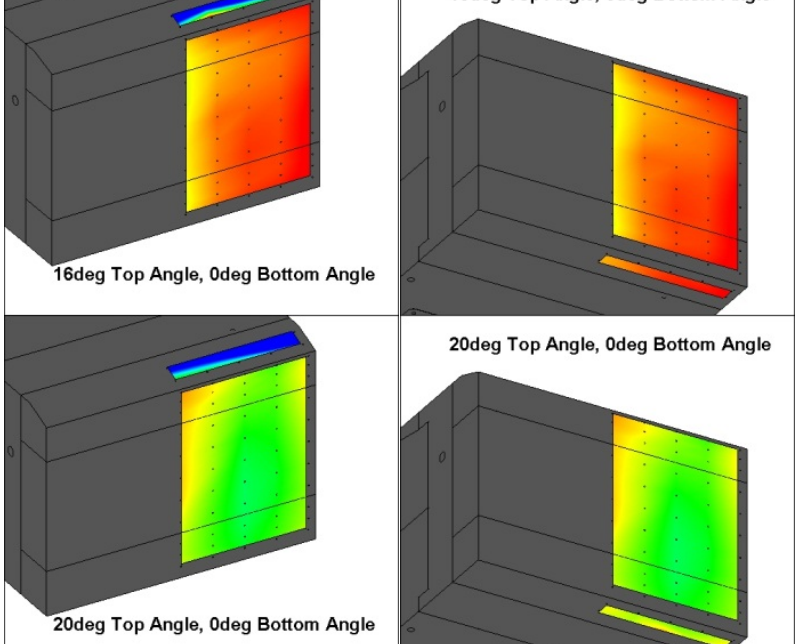

20deg Top Angle, Odeg Bottom Angle

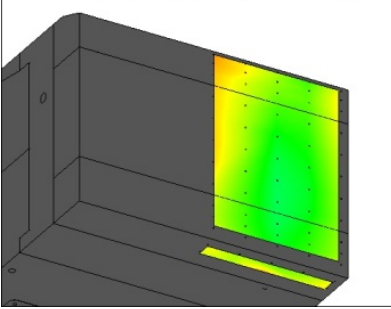

Figure 9 Time Averaged Pressure Distributions on Rear Projecting Surfaces for $0^{\circ}$ bottom taper angles

While the flowfield has not, at this stage, been measured directly, the base pressure data indicates a change to the size, shape and relative location of the wake torus structure. It is known from the work of Littlewood \& Passmore [13] that including a rear slant angle encourages a balancing of the torus in the vertical plane at the centerline whilst the upper lobe of the torus is also pushed downstream effectively tilting the overall structure. However as a highly three-dimensional flow structure, it cannot be assumed that the slant only creates two-dimensional effects. From the results here, the 
introduction of the top slant appears to be changing the size and strength of the vertical side lobes of the torus and it is this that is the primary mechanism controlling the base pressure.

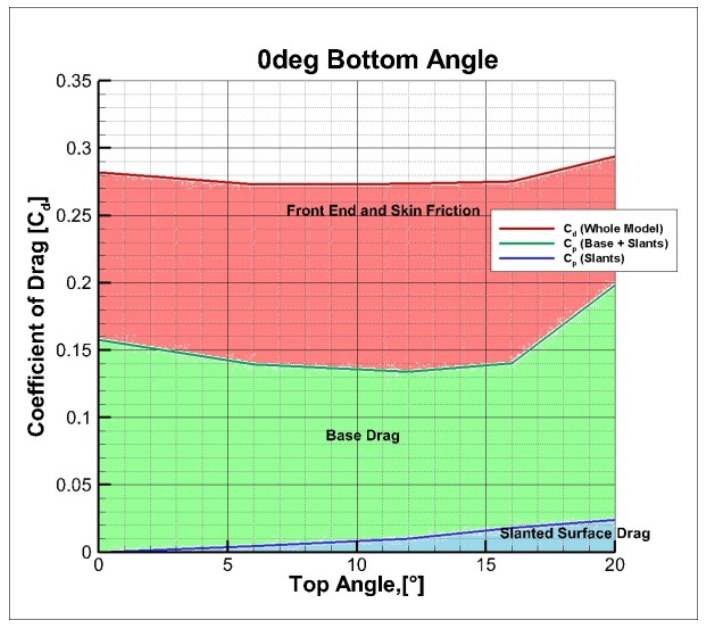

Figure 10 Comparison of contributions to the overall drag coefficient from the rearward projected regions for a $0^{\circ}$ bottom angle

On the slanted surface there is a progressive trend towards flow separation. Starting at $6^{\circ}$ the flow remains attached, seen as a pressure recovery over the length of the slant. The pressure is far lower than for the squareback case though and hence the lift increase seen in the balance data. As the top slant angle increases up to $16^{\circ}$, the lift continues to increase by $\Delta \mathrm{C}_{\mathrm{L}}=0.054$ and then a further $\Delta \mathrm{C}_{\mathrm{L}}=0.040$. While the $12^{\circ}$ case shows evidence of strong suction and therefore flow separation at the top of the rear slant followed by reattachment characterized by pressure recovery, the $16^{\circ}$ sees the separated region cover more of the slant length with possible reattachment within the slant length yielding what would likely be an unsteady flow phenomenon with intermittent separation and reattachment.

For the $20^{\circ}$ top slant angle, the drag increases by a further $\Delta \mathrm{C}_{\mathrm{D}}=0.020$ and the lift reduces by a further $\Delta \mathrm{C}_{\mathrm{L}}=-0.044$ compared to the $16^{\circ}$ top angle configuration. The pressures show far greater suction at the outer edge of the top slant than for any other configuration, indicating the presence of 3D slant edge vortices. While these will generally induce a local downwash over the slant near the vortex structure, and encourage reattachment of the flow, they are also known to create induced drag $[18,21]$. Due to the short slant length the vortices will remain weaker than in a traditional fastback flow field and the influence of these structures will be localized. Therefore the flow in the central region of the slant will remain fully separated, returning the wake inlet condition to one similar to the baseline squareback case but with increased levels of turbulence. The presence of the large, turbulent squareback wake structure, in combination with the 3D slant edge vortices, results in the high drag coefficient seen in Figure 5. All of these changes culminate in the flow no longer following the previous trend and the deviation from the trend seen in Figure 7 and Figure 8.
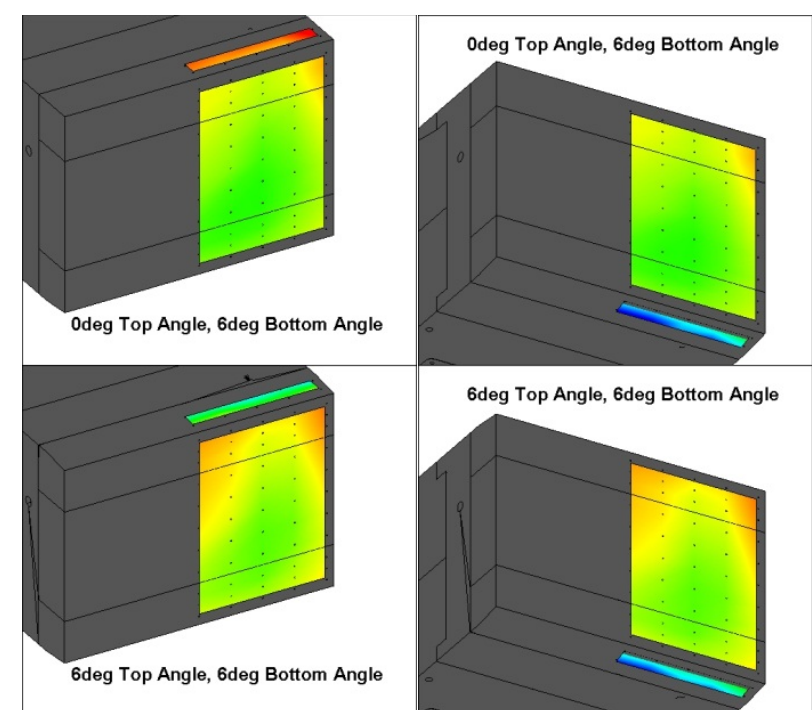

6deg Top Angle, 6deg Bottom Angle

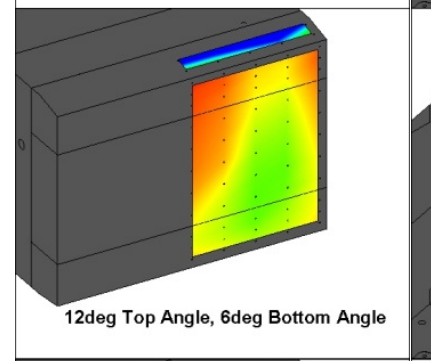

12deg Top Angle, 6deg Bottom Angle
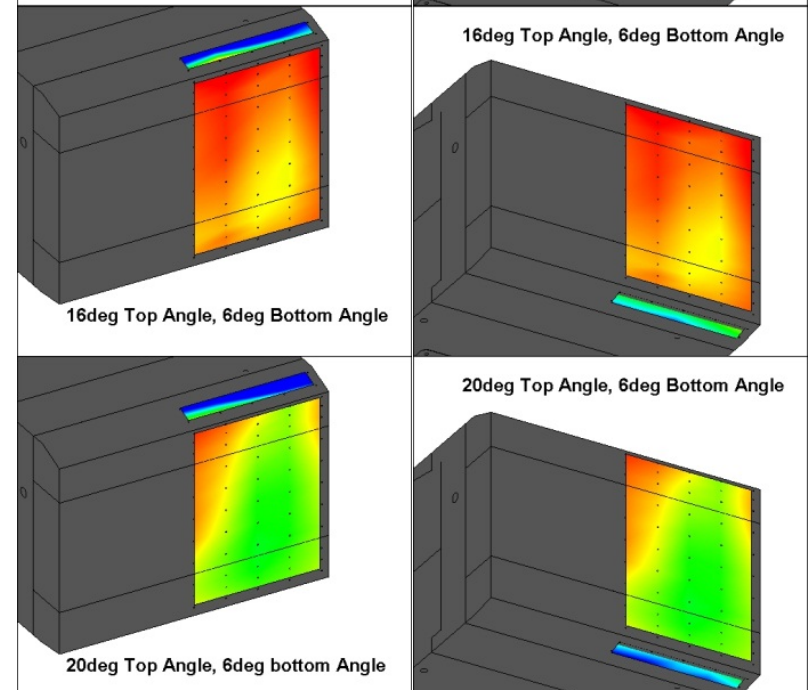

20deg Top Angle, 6deg Bottom Angle
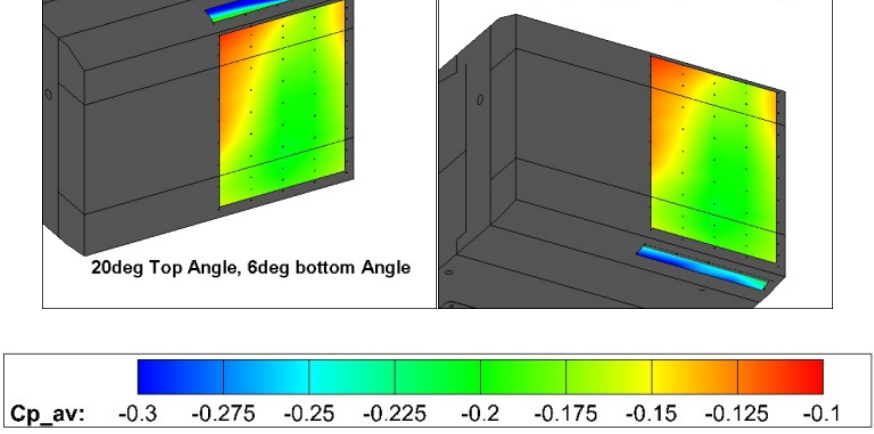

Figure 11 Time Averaged Pressure Distributions on Rear Projecting Surfaces for $6^{\circ}$ bottom taper angles

To reduce this data further the area weighted pressure coefficients of all rear projected surfaces were calculated using Equation 3, and the results are presented in Figure 10 for the $0^{\circ}$ bottom angle case. The individual contribution from each surface can be calculated and the sum of these gives the total pressure drag contribution from the rear end. It is assumed that the fore-body pressure and skin-friction drag contributions are all that remains when the drag of the rear end surfaces has been accounted for; although there will be some error 
due to the drag of the supporting struts and the exposed measurement equipment.

$\overline{C_{P}}=\frac{1}{A} \int_{0}^{A} C_{P} d A$

\section{A-Model Frontal Area $\quad d A-$ Area of influence of each tapping $C_{p}$-Time Averaged Continuity Corrected Pressure Coefficient}

Taking the $0^{\circ}$ bottom angle case, it is seen that increasing top angle results in a linearly increasing drag contribution from the slanted surfaces (in this case only the top angle is contributing). Now that the contributions from the slants have been separated, it is clear that the base pressure with a $16^{\circ}$ top angle is lower than that at $6^{\circ}$, but the slant drag is higher so that the total rear end drag is very close. As the top angle is increased, there are slight changes to the contribution from the fore-body and skin friction, most notably this is reduced for a $20^{\circ}$ top angle, where the base drag contribution is markedly higher.

These results show that with only a small rear end taper some of the advantageous wake characteristics of a fastback geometry can be captured, whilst maintaining the design characteristics of a squareback geometry. The trends are similar to the work of Littlewood \& Passmore [13], however the change in the model ground clearance from $(\mathrm{h} / \mathrm{H})=0.10$ to $(\mathrm{h} / \mathrm{H})=0.17$ affects the minimum drag configuration.

Considering the $6^{\circ}$ bottom angle case, Figure 11, there are few changes to the pressures on the top slant angle compared to the $0^{\circ}$ bottom angle, however the pressure on the diffusing underbody rear taper has dropped significantly and consequently the base pressure distribution is very different. This is characterized by a slightly higher pressure on the upper section around the centerline and an area of higher pressure in the upper outer section. The pressure contours on the diffusing section indicate a small amount of separation occurring at the diffuser inlet but this reattaches, giving pressure recovery towards the trailing edge of the diffuser.

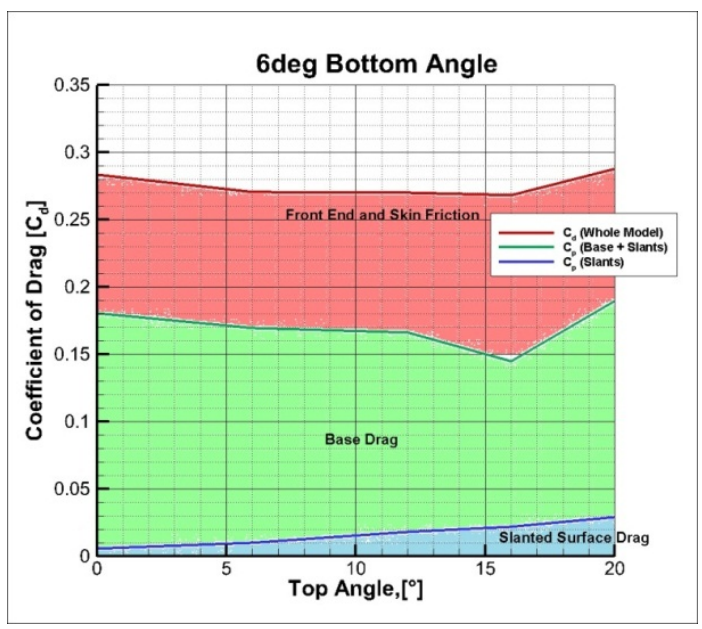

Figure 12 Comparison of contributions to the overall drag coefficient from the rearward projected regions for a $6^{\circ}$ bottom angle

Looking at the contributions of each surface, Figure 12, again there is a linear increase in drag contribution from the slanted surfaces (top and bottom) and although the total drag is very similar for the $0^{\circ}$ and $6^{\circ}$ cases there is an increase in the base drag contribution for the $6^{\circ}$ case suggesting that the small diffuser is having an effect on the front

Page 7 of 11

stagnation and therefore varying the fore-body drag such that the rear drag increase is nullified.
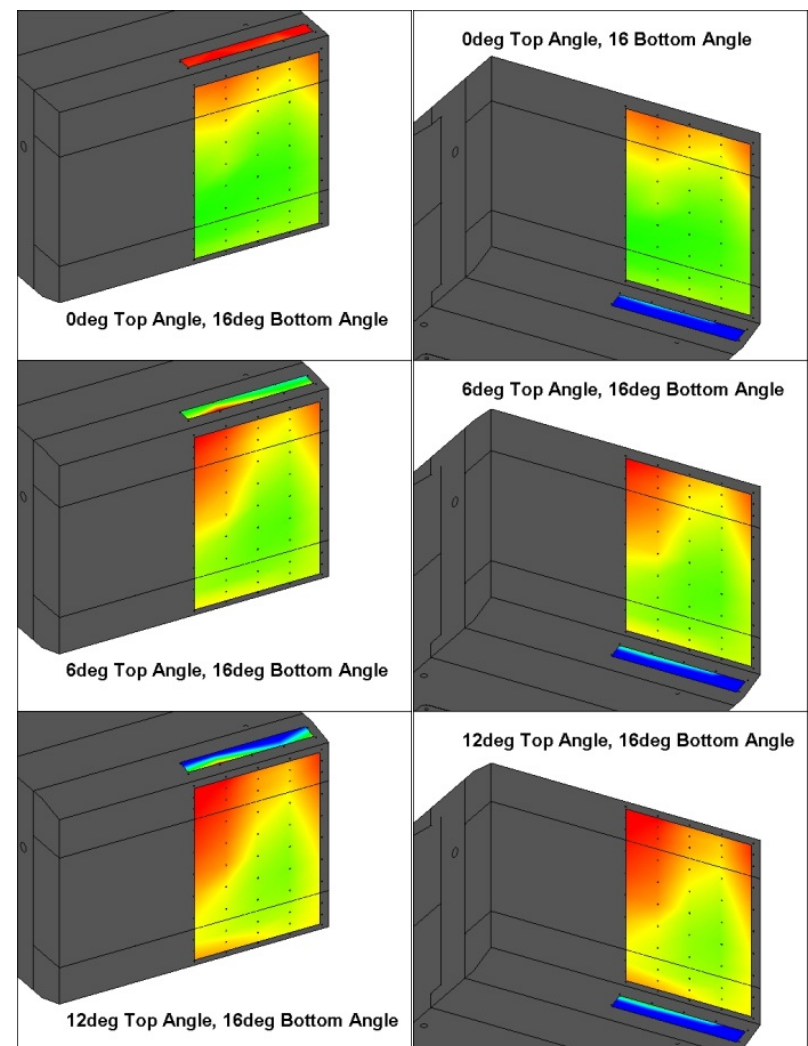

12deg Top Angle, 16deg Bottom Angle .

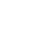


at the bottom of the base. This would also have been expected for the $6^{0}$ diffuser also, but was not seen. However the size of this region is very small and therefore its omission in the $6^{\circ}$ results may be due to insufficient pressure tapping resolution in this region. Above this small pressure recovery is a region of low pressure which is of a similar structure to the $6^{\circ}$ diffuser however it is evidently stronger as it is less influenced by the increasing top angles. The top angle does create a pressure recovery in the upper central and upper outer regions of the base, which increases between $0^{\circ}$ to $16^{\circ}$ before the flow separation on the $20^{\circ}$ top angle reduces this effect back to near baseline conditions.

The relative contributions to drag coefficient, Figure 14, show a far more consistent fore-body drag contribution with a reducing base drag with increasing top angle up to $16^{\circ}$.

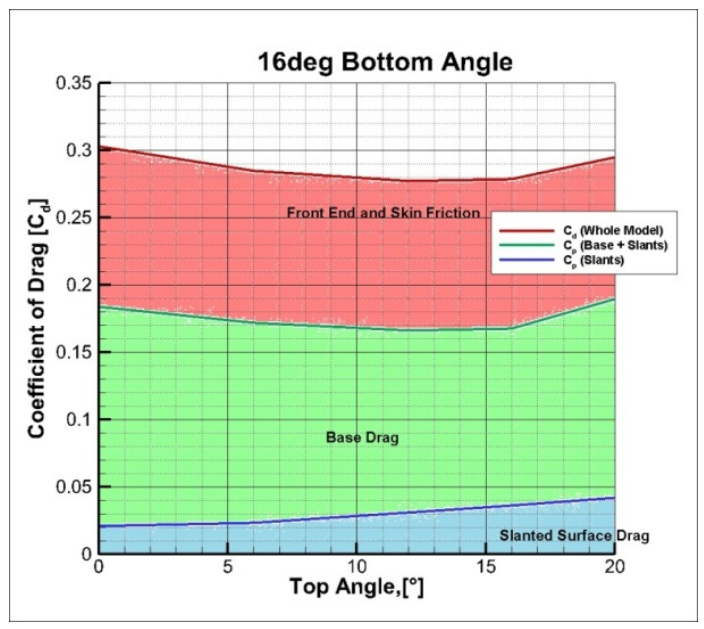

Figure 14 Comparison of contributions to the overall drag coefficient from the rearward projected regions for a $16^{\circ}$ bottom angle

\section{Side Edge Tapers}

Applying similar tapers to the model sides at angles of $0^{\circ}, 4^{\circ}, 8^{\circ}, 12^{\circ}$, $16^{\circ}$ and $20^{\circ}$, balance and surface pressure results were taken at $0^{\circ}$ yaw to understand how the wake mechanisms are changed with inwash from the model sides.

The drag results are presented in Figure 15, and show a parabolic trend; the drag initially decreases as the side slant angle is increased from $0^{\circ}$ with minimum drag occurring at a $12^{\circ}$ side angle with $\Delta \mathrm{C}_{\mathrm{D}}=-$ 0.020 from the baseline squareback case. This is similar in magnitude to the drag change between baseline and optimum in the top and bottom slant work. Above $12^{\circ}$ the drag increases more quickly and for the $20^{\circ}$ slant angle the result is close to that of the baseline squareback case.

The time averaged surface pressure results, Figure 16, show an increasing base pressure from the baseline squareback case for the $4^{\circ}$, $8^{\circ}$ and $12^{\circ}$ cases, evidently lowering the drag. For the $16^{\circ}$ and $20^{\circ}$ slant angle cases there is significant pressure recovery in the upper half of the base while a region of suction is developing in the lower half, lowering the average base pressure. This change in the wake flow topology corresponds with the increasing drag seen in Figure 15.

Page 8 of 11
On the side slant, the surface pressures show fully attached flow for $4^{\circ}$ and $8^{\circ}$, at angles greater than $8^{\circ}$ there is a suction peak on the leading edge of the slant followed by pressure recovery over the slant length indicating reattachment.

In the side slant surface pressures of the $12^{\circ}, 16^{\circ}$ and $20^{\circ}$ configurations the slant edge longitudinal vortices are evident in the reduced pressure at the top and bottom. These encourage the flow to remain attached in a similar fashion to the slant edge vortices presented in the previous section.

The base pressure increase seen between $4^{\circ}$ to $12^{\circ}$ comes mainly from a higher pressure region encroaching from the model sides, extending to the model centerline for the $12^{\circ}$ configuration. This again indicates a strengthening of the sides of the torus structure and an increase in its relative size; this would be expected to be a similar mechanism to increasing the downwash on a single top slant giving an increase in size to the upper vortex structure.

Between the baseline squareback case and the $4^{\circ}$ side angle, there is significant pressure recovery to the upper outer quadrant of the base, and the lower outer base quadrant loses some pressure giving a more balanced wake. However for the $16^{\circ}$ and $20^{\circ}$ side angle cases, there is a reduction in pressure over the lower central quadrant of the base indicating that there may now be some unsteady flow separation reducing the strength of the in-wash.

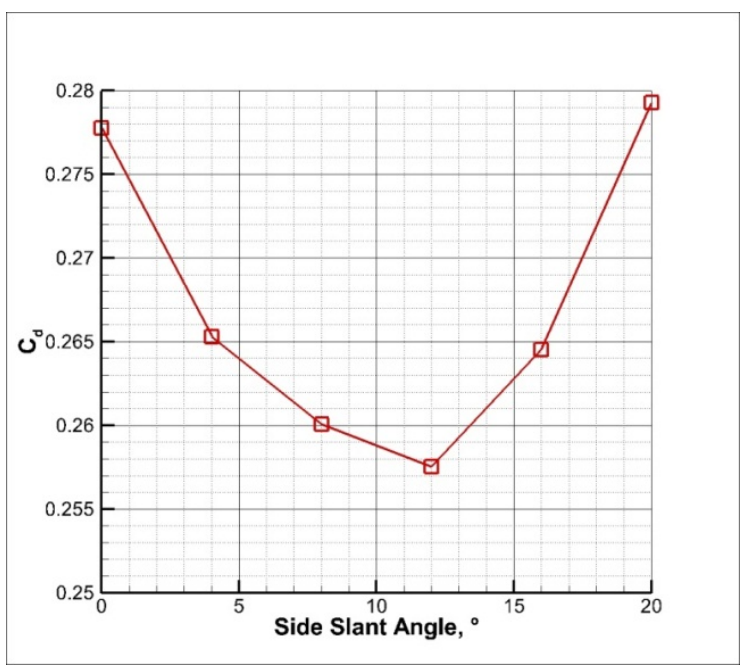

Figure 15 Drag Coefficient for all side angles at $0^{\circ}$ Yaw

\section{Summary / Conclusions}

The work conducted here has used balance and surface pressure measurements to gain an understanding of the flow field interactions of short rear end tapers on a simplified vehicle geometry.

- $\quad$ For top and bottom edge tapers the optimum drag result is obtained with a top edge taper of $16^{\circ}$ and a bottom edge taper of $6^{\circ}$ with a $\Delta \mathrm{C}_{\mathrm{d}}=-0.014$ compared to the baseline squareback case. Applying top and bottom tapers asynchronously, the interactions between the up-wash and down-wash has a large effect on the base pressure distribution and also the separation seen in the underbody region. 
- $\quad$ By increasing the top slant angle the pressures infer that there is an increase in the size of the upper lobe of the wake torus, but also an increase in the size of the torus sides and it is the latter that is the primary base pressure recovery mechanism.

- $\quad$ For the side taper work, the optimum drag result occurs at a $12^{\circ}$ angle with a $\Delta C_{D}=-0.020$.

- $\quad$ For the side taper work, increasing the side angle up to $12^{\circ}$ increases size of the side lobes of the wake torus which again gives the base pressure recovery from the model sides; this creates a balanced wake structure in the vertical plane.
- When the side taper angle is increased to $16^{\circ}$ and beyond, there is a change to the wake topology resulting in an imbalance of the toroidal vortex structure, giving dominance to the upper and lower lobes, and a rapid increase in the model drag.

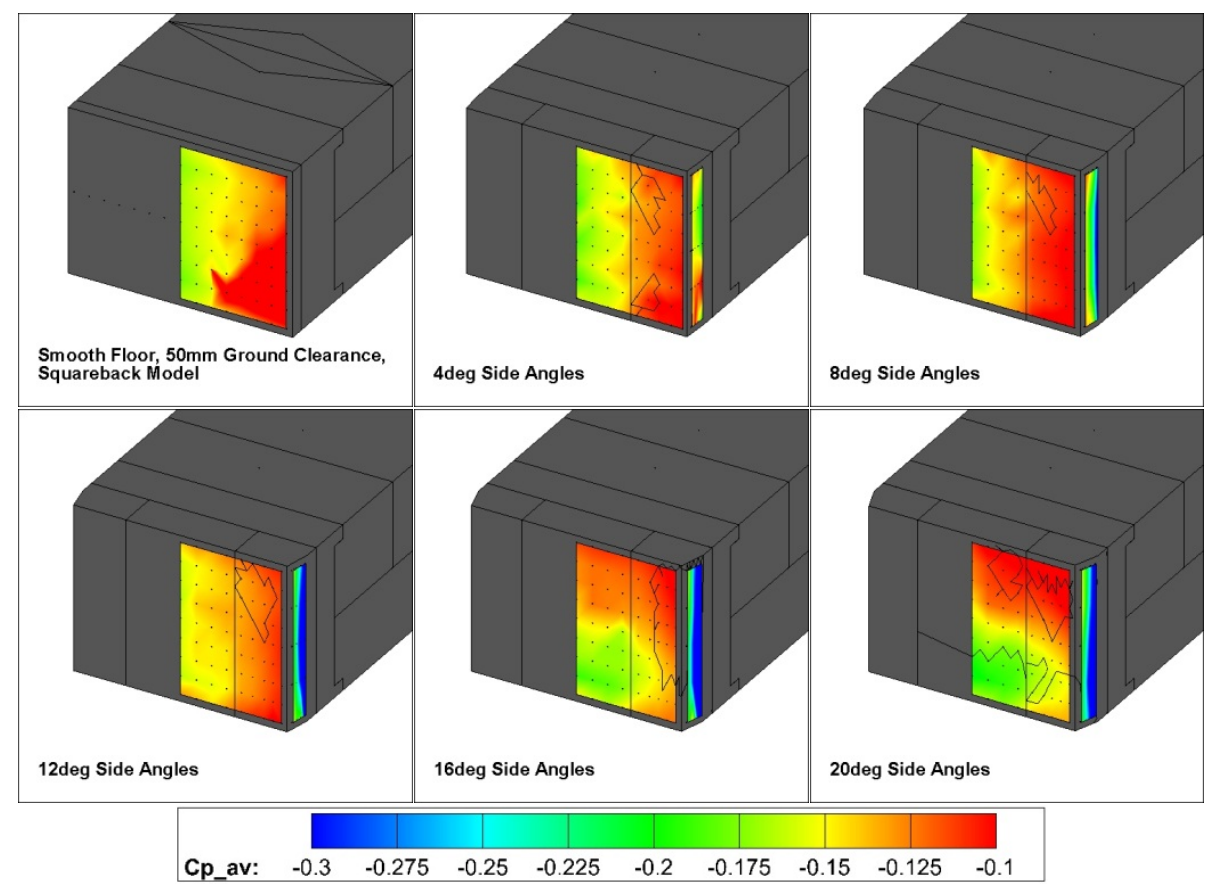

Figure 16 Time averaged surface pressure contour plots for all side taper angles

The next stage in this work is to use particle image velocimetry to examine the changes to the near and far wake, particularly focusing on the interactions and wake shape changes created by the top and bottom tapers as well as changes to the wake width caused by flow entrainment from the model sides.

Further experimental work applying these short slants to all of the base edges simultaneously but asynchronously is planned; this will investigate the effects the encroachment of flow from the side angles has on the up- and downwash for the top and bottom slant angles.

\section{References}

1. Gurlek, C., Sahin, B., Memduh Ozhan, G., "PIV studies around a bus model”, Experimental Thermal and Fluid Science, Vol 38, pp 115-126, 2012.

2. Beaudoin, J., Aider, J., "Drag and Lift Reduction of a 3D bluff body using flaps”, Experiments in Fluids, Vol 44, pp491-501, 2008.

3. McCallen, R., Salari, K., Ortega, J., Castellucci, P., et al., “DOE’s Effort to Reduce Truck Aerodynamic Drag
Through Joint Experiments and Computations”, SAE Technical Paper 2005-01-3511, 2005.

4. Howell, J., Sims-Williams, D., Sprot, A., Hamlin, F., Dominy, R., "Bluff Body Drag Reduction with Ventilated Base Cavities”, SAE Int. J. Passenger Cars - Mech. Syst., Vol 5(1), 2012.

5. Lanser, W., Ross, J., Kaufman, A., “Aerodynamic performance of a drag reduction device on a full scale tractor/trailer”, SAE Technical Paper 912125, 1991.

6. $\quad$ Aider, J., Beaudoin, J., Wesfreid, J.E., "Drag \& Lift Reduction of a 3D bluff-body using Active Vortex Generators”, Exp. Fluids, Vol 48, pp 771-789, 2010.

7. Englar, R., "Drag Reduction, Safety Enhancement, and Performance Improvements for Heavy Vehicles \& SUVs Using Advance Pneumatic Aerodynamic Technology”, SAE Technical Paper 2003-01-3378, 2003.

8. Joseph, P., Amandolese, X., Aider, J., "Drag Reduction on the $25^{\circ}$ slant Angle Ahmed reference body using pulsed jets”, Exp. Fluids, Vol 52(5), pp 1169-1185, 2012. 
9. Roumeas, M., Gillieron, P., Kourta, A., "Drag Reduction by Flow Separation Control on a Car Afterbody”, International Journal for Numerical Methods in Fluids, Vol 60, pp 1222-1240, 2009.

10. Irving Brown, Y.A., Windsor, S., Gaylard, A.P., "The effect of Base Bleed and Rear Cavities on the Drag of an SUV”, SAE Technical Paper 2010-01-0512, 2010.

11. Howell, J., Sheppard, A., Blakemore, A., “Aerodynamic Drag Reduction for a Simple Bluff Body Using Base Bleed”, SAE Technical Paper 2003-01-0995, 2003.

12. Ahmed, S.R., Ramm, G., Faltin, G., "Some Salient Features of the Time-Averaged Ground Vehicle Wake”, SAE Technical Paper 840300, 1984.

13. Littlewood, R., Passmore, M., "The Optimisation of Roof Trailing Edge Geometry of a Simple Squareback”, SAE Technical Paper 2010-01-0510, 2010.

14. Littlewood, R., Passmore, M., Wood, D., “An Investigation into the Wake Structure of Squareback Vehicles and the Effect of Structure Modification on the Resultant Vehicle Forces”, SAE Technical Paper 2011-37-0015, 2011.

15. Grandemange, M., Mary, A., Gohlke, M., Cadot, O., "Effect on drag of the flow orientation at the base separation of a simplified blunt road vehicle”, Exp Fluids, Vol 54, pp1529, 2013.

16. Johl, G., Passmore, M., Render, P., "Design Methodology and Performance of an indraft wind tunnel”, The Aeronautical Journal, Vol 108(1087), pp465-473, 2004.

17. Howell, J.P., Hickman, D., “The Influence of a Ground Simulation on the Aerodynamics of Simple Car Shapes with an Underfloor Diffuser”, SAE Technical Paper 970134, 1997.

18. Howell, J., Le Good, G., "The effect of backlight aspect ratio on Vortex \& Base drag for a simple car-like shape”, SAE Technical Paper 2008-01-0737, 2008.

19. Jowsey, L., Passmore, M., "Experimental study of multiple-channel automotive underbody diffusers", Proceedings of The Institution of Mechanical Engineers Part D - Journal of Automobile Engineering, vol 224, pp865-879, 2010.

20. Cooper, K., Bertenyi, T., Dutil, G., Syms, J., Sovran, G., "The Aerodynamic Performance of Automotive Underbody Diffusers”, SAE Technical Paper 980030, 1998.

21. Howell, J., Le Good, G., "Vortex Drag for a Simple CarLike Shape", $5^{\text {th }}$ MIRA International Vehicle Aerodynamics Conference, $13^{\text {th }}$ October 2004.

\section{Contact Information}
A-K. Perry:
01509227263
M. Passmore
A.Perry@lboro.ac.uk
M.A.Passmore@lboro.ac.uk

\section{Acknowledgements}

The authors would like to thank Rob Hunter for his work manufacturing the different model geometries for testing.

Page 10 of 11 


\section{Appendix}

\section{Time Averaged Base Pressure Plots for all Top and Bottom Angle Slant Angle Configurations}

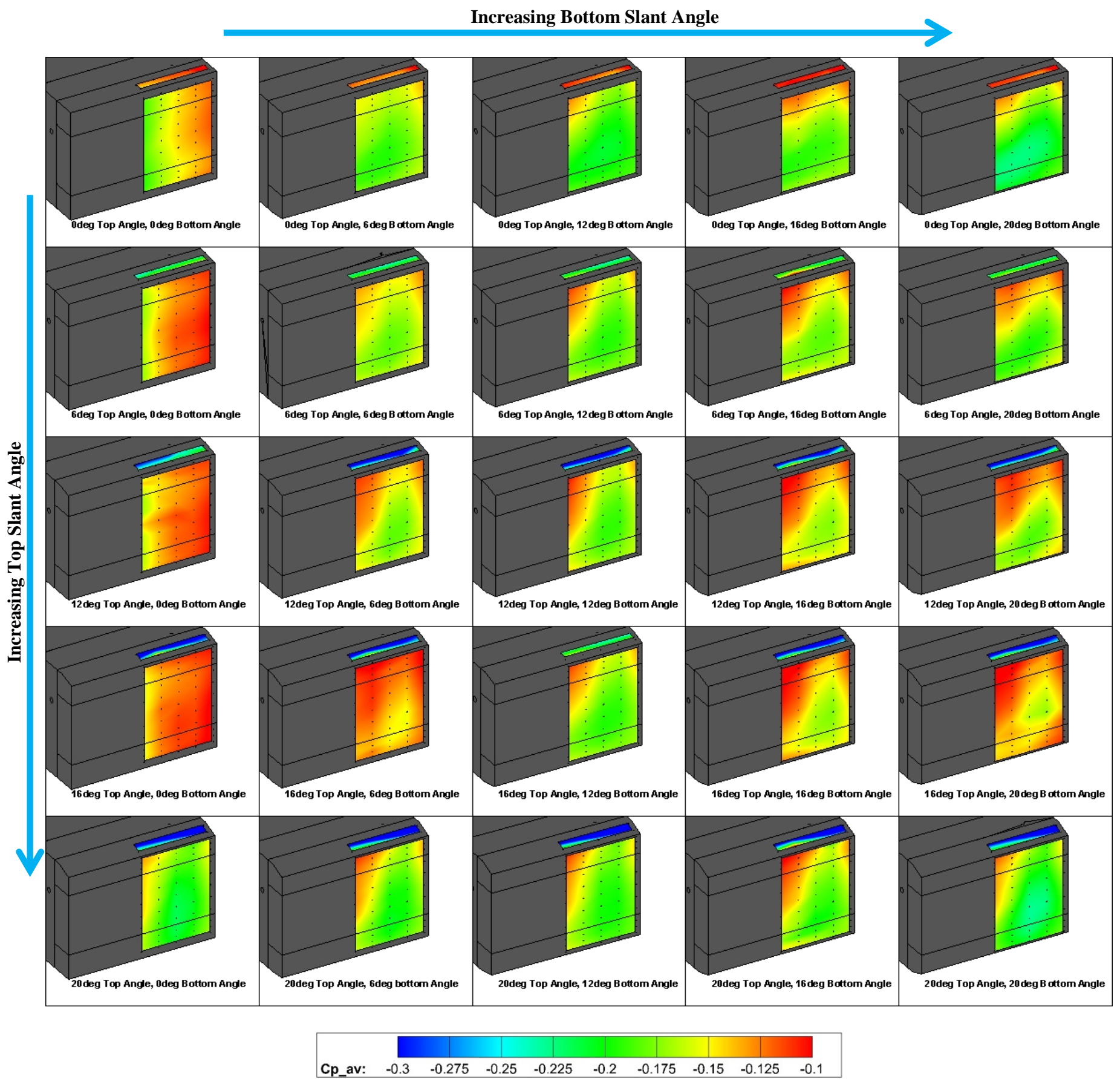

Page 11 of 11 